\title{
Evidence of butyl benzyl phtalate induced modifications in a model system developed in vitro
}

\author{
M.G. Sabbieti ${ }^{1}$, L. Marchetti ${ }^{1}$, R. Curini ${ }^{2}$, G. Menghi ${ }^{1}$, A. Roda ${ }^{3}$, M.V. Russo ${ }^{4}$, \\ C. Nugnes ${ }^{2}$ and S. Materazzi ${ }^{2 *}$ \\ ${ }^{1}$ Dep. of Comparative Morphological and Biochemical Sciences, University of Camerino, Italy \\ 2 Dep. of Chemistry, University "La Sapienza", p. le A. Moro 5, 00185 Roma, Italy \\ ${ }^{3}$ Dep. of Chemistry, University of Bologna, Italy \\ ${ }^{4}$ DISTAAM, University of Molise, Campobasso, Italy
}

\begin{abstract}
The phtalate induced modifications on osteoblastic cells have been monitored in a model system in vitro: preliminary encouraging results, independently obtained by calorimetry (DSC), confocal laser scanning microscopy (CLSM) and coupled gas chromatography-mass spectrometry (GC-MS), show the possibility to relate kinetic data and immunofluorescence images, that are very useful to show the entry and the localization of phtalate into the cells. The calorimetric data were obtained by a cellular living system growth and reacting into the aluminium pans.
\end{abstract}

Key words. Butyl benzyl phtalate - rat osteoblasts - CLSM - DSC - GC-MS.

\section{Introduction}

Phtalic acid diesters, commonly known as phtalates, are a group of organic chemicals that have a variety of industrial uses, including use as plasticisers in a wide range of household and consumer goods, uses in lubricating oils and uses as carriers for perfumes in cosmetics.

The use of phtalates is restricted, for example, to the manufacture of materials such as some adhesives and some printing inks [1]. Phtalates are no longer used in the manufacture of cling film and most other food contact plastic materials [2].

The release of phtalates into the environment may occur during the production and distribution of phtalates, during the manufacture or the use of phtalate-based products, or after the waste of these products.

As a result of their extensive use and their moderate resistance to degradation, phtalates are widely distributed in the environment and are often found at low levels in food $[3,4]$.

After 30 years of phtalate production, the toxic potential of these substances was recognized in the early 70's [5], but the actual growing problem is their potential activity as endocrine disrupting compounds (EDC) [6-9]; benzyl, butyl and di-n-butyl phtalates have been supposed EDC with estrogenic properties [10-12].

The aim of this study was to prove the phtalate induced modifications in cells by monitoring the butyl benzyl phtalate (BBP) effect as a function of the time; by confocal laser scanning microscopy (CLSM), differential scanning calorimetry (DSC) and coupled gas chromatography-mass spectrometry (GC-MS), the effect of BBP in rat osteoblastic cells is enhanced. The calorimetric data were obtained by a living system growth and reacting into the aluminium pans, exactly reproducing the situation observed by laser microscopy images.

\section{Experimental}

The in vitro experimental conditions, used to obtain the CLSM images, were exactly reproduced into aluminium pans to relate the calorimetric signal and the effective phtalate action. Moreover, for GC-MS analysis cell pellets were obtained from parallels cultures.

\section{Cell culture for DSC experiment}

The presently employed culture system was planned for the first time by adapting the original method [13].

Cells were plated on aluminium pans, previously cleaned and sterilized, and introduced on 6-well culture dishes (Corning). Cells were growth to confluence in F12 culture medium (Gibco) containing $5 \%$ FBS penicillin and streptomycin; the effective presence of cells into the pans was tested by staining with haematoxylin-eosin and observing them under a stereomicroscopy.

When cells were confluent, in one aluminium pan the butyl benzyl phtalate (BBP) was added at a concentration of

*Correspondence and reprints.

Received August 1, 2000; revised September 26, 2000; accepted October 5, 2000. 


\section{Original articles}

$10^{-6} \mathrm{M}$, using $95 \%$ ethanol as vehicle; this pan was introduced in the sample furnace, while the other pan without BBP addition was placed in the reference furnace; the DSC isothermal record was immediately started.

\section{DSC analysis}

The DSC curves were obtained using a Perkin Elmer DSC7, in isothermal mode, at $20{ }^{\circ} \mathrm{C}$ for 60 minutes.

\section{Baseline}

In both the reference and the sample furnaces, the aluminium pans with inside the cells, the culture medium and the antibiotics were placed; the resulting DSC trace was used as the baseline and automatically subtracted.

\section{Isothermal scan}

In the reference furnace, an aluminium pan with inside the cells, the culture medium and the antibiotics was placed, while in the sample furnace an aluminium pan with inside the cells, the culture medium, the antibiotics and $10 \mu \mathrm{L}$ of a solution of benzyl-butyl-phtalate (final-concentration $\left.10^{-6} \mathrm{M}\right)$ was placed.

\section{Cell culture for confocal analysis}

Immortalized rat osteoblastic Py1a cells (kindly provided by prof. Hurley, University of Connecticut, Farmington, USA) were plated on coverslips, previously cleaned and sterilized, on 6-well culture dishes and were growth with F12 culture medium (Gibco) added with $5 \%$ fetal bovine serum (FBS), penicillin and streptomycin.

When the cells were significantly confluent (80\%), they were treated with butyl benzyl phtalate (BBP) at a concentration of $10^{-6} \mathrm{M}$ using $95 \%$ ethanol as vehicle, for $10 \mathrm{~min}$ utes and 60 minutes, as suggested by DSC profile.

\section{Immunostaining}

After treatment with BBP, cells were fixed with a $4 \%$ paraformaldehyde in phosphate buffer saline solution (PBS),

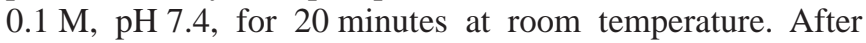
rinsing, cells were incubated with an antibody that selectively recognizes o-phtalate esters [14] for 2 hours at room temperature. Cells were then rinsed in PBS and incubated with the secondary antibody goat anti-rabbit conjugated with fluorescein isothiocyanate (FITC) (Sigma) diluted 1:75 for 2 hours at room temperature. Staining controls were carried out using a nonimmune rabbit immunoglobulin $\mathrm{G}$.

After a final washing, the coverslips were mounted on slides with PBS/glycerol (1:1).

\section{CLSM analysis}

Specimens mounted on glass slides were viewed with a confocal laser scanning microscope (Bio-Rad MRC 600, BioRad, Hertfordshire, UK) equipped with a krypton/argon laser. Microphotographs were taken by PIC format file and printed with Epson Stylus photo 750 on Epson glossy photo paper.

\section{GC-MS analysis}

Simultaneously growth cells were centrifugated and collected in pellets (control as reference, after 10 minutes and after 60 minutes from the BBP administration); the phtalate extraction was carried out by hexane (3 extractions) after a treatment of each cell pellet with a $1 \mathrm{M}$ sodium chloride solution. The hexane solution was injected in a HewlettPackard gaschromatograph, model 5890 series II, coupled to a HP 5970B mass selective detector (fused-silica CP-sil 8 CB capillary column, $25 \mathrm{~m}$ lenght, $150 \mu \mathrm{m}$ ID, $\mathrm{d}_{\mathrm{f}}=0.12 \mu \mathrm{m}$ by Chrompak). The column was kept at $40{ }^{\circ} \mathrm{C}$ for 5 minutes and heated up to $270{ }^{\circ} \mathrm{C}$ at a programmed temperature rate of $10{ }^{\circ} \mathrm{C} \mathrm{min}-1$; injections were made in splitless mode (30 sec delay before opening the splitter) using helium gas as carrier at $u=36 \mathrm{~cm} \mathrm{sec}^{-1}$.

\section{Results}

The DSC curve resulting from the subtraction of the baseline (curve $1 \mathrm{~b}$ in figure 1 ) from the phtalate-added sample is reported in the figure 1a. This curve shows a first endothermic process with a maximum at 9 minutes, overlapped by a second more enhanced endothermic process that reaches a steady state after 50 minutes.

CLSM analysis was found to represent a very useful approach to investigate the location of BBP within the rat osteoblastic cells Pyla and to follow the internalization patterns. In the figure $2 \mathrm{a}$, it is shown the basal BBP labeling in untreated cells. After 10 minutes, it can be observed the BBP immunostaining to slightly increase in the cytoplasm (Fig. 2b). Between 20 minutes and 1 hour, there is the most intense labeling around the nucleus (Fig. 2c).

The GC-MS analysis shows a high concentration of BBP in the cell pellet hexane extracting solution after 10 minutes (about $80 \%$ of the initial amount), while the control and the 60 minutes hexane extracting solutions show only traces of the butyl benzyl phtalate (Fig. 3).

\section{Discussion}

The endocrine-disrupting action of phtalates in living organisms is an evermore growing problem; few studies however really prove the real impact of these frequently used plasticizers on biota.

The possibility to monitor the in vitro reactions induced by a substance on living cellular systems is very useful to compare the kinetic informations with the images obtained by immunochemistry in situ starting from the same systems processed by histochemistry. 


\section{Original articles}

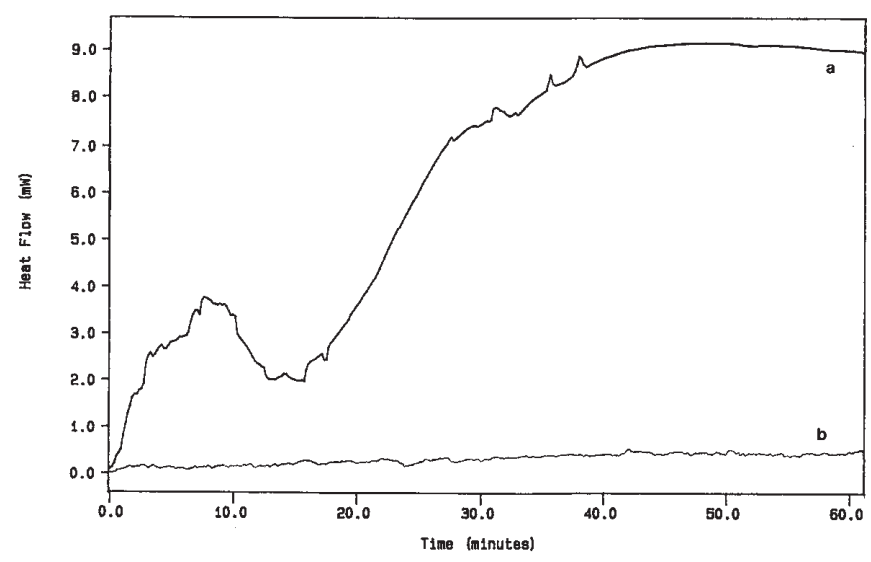

Figure 1. a) DSC curve of Pyla osteoblastic rat cells under benzyl butyl phtalate administration; b) DSC reference curve of Py1a osteoblastic rat cells. Closed pans, isothermal mode at $20{ }^{\circ} \mathrm{C}$ $\min ^{-1}$.

Goal of this study was to relate the informations independently obtained by DSC, CLSM and GC-MS techniques on rat osteoblastic cells under exposure to phtalate solutions; for this purpose, the in vitro experimental conditions used to obtain the CLSM images were exactly reproduced into aluminium pans to relate the calorimetric signal and the effective phtalate action and cell pellets were collected from the same parallel 6-well culture dishes.

Looking at the DSC trace and to the CLSM images, it can be clearly seen that the two different techniques give a clear characterization of the system that undergoes clear modifications.

Ten minutes after the benzyl butyl phtalate addition, the CLSM image enhances the entry into the cell by weak fluorescence, with the corresponding endothermic peak in the DSC curve having a maximum at 9 minutes (see figure 1 and figure $2 b$ ). This peak is however overlapped by the second endothermic DSC process that describes the increasing energy need of the reaction, reaching a steady state at 60 minutes. At the same time, the CLSM images show an increasing fluorescence in the cell cytoplasm with a maximum at 60 minutes and a localization around the nucleus (Fig. 2c).

The energy balance and the increasing of the fluorescence into the cells give the experimental evidence of a clear reaction happening into the system, thus suggesting a sequence of phtalate-induced modifications, typical of endocrine-disrupting agents, with the supposed production of metabolites.

This hypothesis is confirmed by the preliminary GC-MS data obtained on cell pellets simultaneously growth: in the control pellet extracting solution there are obviously only traces of BBP, while in the pellet extracting solution after 10 minutes from the phtalate addition there is about $80 \%$ of the BBP added amount, showing the entry of the phtalate into the cells and confirming the CLSM image (Fig. 3).
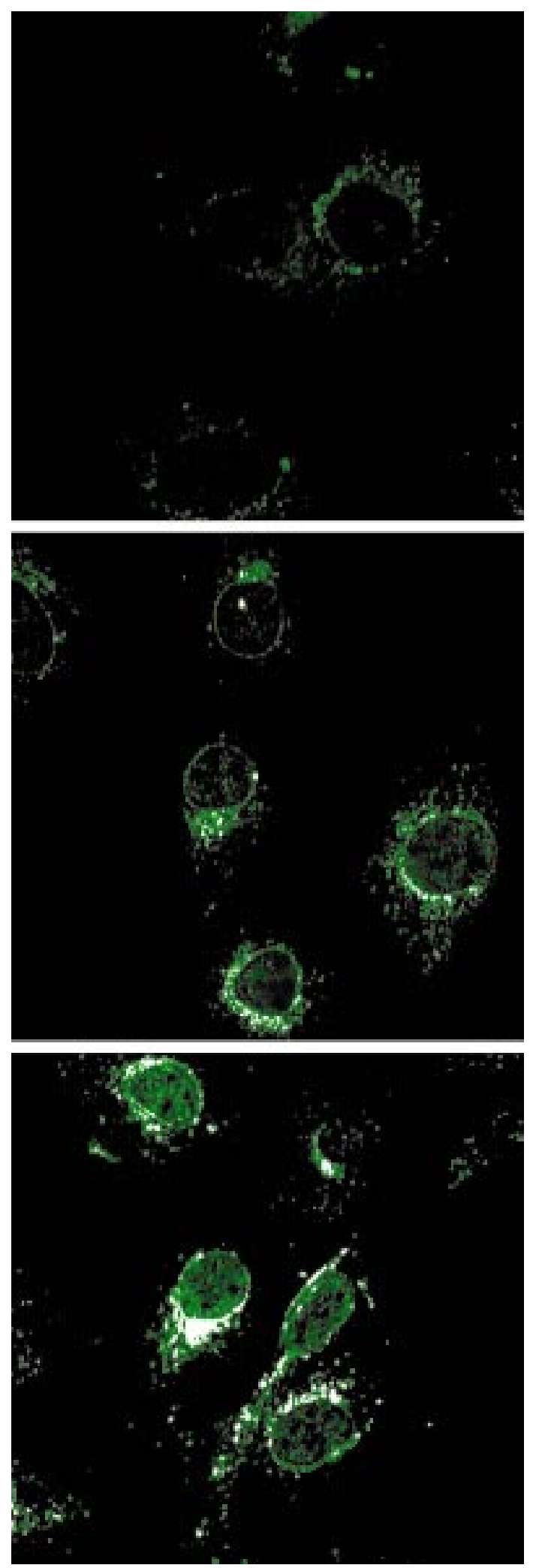

Figure 2. CLSM images of Pyla osteoblastic rat cells under benzyl butyl phtalate administration: a) basal BBP labeling in untreated cells; b) 10 minutes immunostating; c) 60 minutes immunostating 


\section{Original articles}

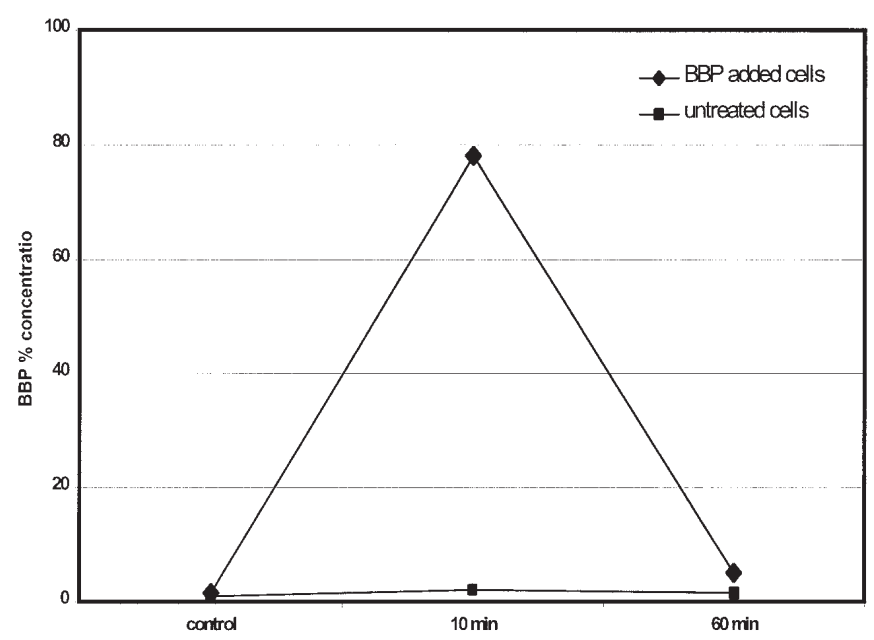

Figure 3. Butyl benzyl phtalate concentration in the cell pellets (GC-MS analysis).

There are again only traces of phtalates in the pellet extracting solution after 60 minutes from the phtalate addition, this suggesting the possibility of the cell induced reaction to metabolise the BBP in conjugated derivatives: the conjugation allows the imaging on the CLSM photos, but the different molecular weight results in the lack of the characteristic $\mathrm{m} / \mathrm{z}$ values of the BBP $(\mathrm{m} / \mathrm{z}=91,149,206,238$, 312) and of the corresponding peak on the GC-MS chromatogram.

These preliminary encouraging results, obtained by DSC, CLSM and coupled GC-MS techniques, relating kinetic data and immunofluorescence images, are very useful to show the localization of BBP; work is currently in progress and the investigation will be continued to characterize the metabolic products and to test the different answers as a function of different concentration or exposure, and of different cellular systems.

\section{Acknowledgements}

This work was supported by Grant MURST $40 \%$ from the Italian Ministry of University and Scientific Research.

\section{References}

1. Ministry of Agriculture, Fisheries and Food, Food Surveillance Information sheet 60, 1995.

2. L. Castle, L.; Gilbert, J.; Eklund, T. Food Addit.Contam. 1990, 7, 591 .

3. Sharman, M.; Read, W.A.; Castle, L.; Gilbert, J. Food Addit. Contam. 1994, 11, 375.

4. Cerbulis, J.; Byler, D.M. J. Agric. Food Chem., 1986, 34, 198.

5. Mayer, F.L.; Stalling, D.L.; Johnson, J.L. Nature 1972, 238, 411.

6. Gilbert, J. The Science of the Total Environment 1994, 143, 103.

7. Sharpe, R.M.; Fisher, J.S.; Millar, M.M.; Jobling, S.; Sumpter, J.P. Environm. Health Perspectives 1995, 103, 1136.

8. Jobling, S.; Reynolds, T.; White, R.; Parker, M.G.; Sumpter, J.P. Environm. Health Perspectives 1995, 103, 582.

9. Preziosi, P. Pure Appl. Chem. 1998, 70, 1617.

10. Longnecker, M.P.; Rogan, W.J.; Lucier, G. Annu. Rev. Public Health 1997, 18, 211.

11. Safe, S.H. Ann. Rev. Pharmacol. Toxicol. 1998, 38, 121.

12. Guillette, L.J. jr.; Guillette, E.A. Tox. Ind. Health 1996, 12, 537.

13. Sabbieti, M.G.; Marchetti, L.; Abreu, C.M.; Hand, A.R.; Raisz, L.G.; Hurley, M.M. Endocrinology 1999, 140, 434.

14. Ius, A.; Bagicalupo, M.A.; Meroni, G.; Pistillo, A.; Roda, A. Fresenius J. Anal. Chem. 1993, 345, 345. 